\title{
Stress-Strain Behaviour of Bacterial Concrete Incorporated With Sugarcane Fibres
}

\author{
Ms. P.Kala ${ }^{a}$, Mrs. N. Sivakami ${ }^{b}$, Dr. R. Angeline Prabhavathy ${ }^{\text {c }}$, Dr.Jessy Rooby ${ }^{d}$ \\ ${ }^{a}$ Research scholar, Department of Civil Engineering, Hindustan Institute of Technology and Science. \\ ${ }^{\mathrm{b}}$ Head of Department, Department of Civil Engineering, Government polytechnic college, Karaikudi. \\ c. Professor, Department of Civil Engineering, Hindustan Institute of Technology and Science. \\ ${ }^{\mathrm{d}}$ Head of Department, Department of Civil Engineering, Hindustan Institute of Technology and Science
}

Article History: Received: 10 November 2020; Revised 12 January 2021 Accepted: 27 January 2021; Published online: 5 April 2021

\begin{abstract}
Bacterial concrete is one of the methods of rectifying the micro-cracks developed in the structural elements made of concrete. The gram-positive type bacteria Bacillus subtilis when acquainted with concrete produces calcite precipitation which heals the micro cracks in the concrete. Bacillus subtilis was used with a cell concentration of 106. The optimised percentage replacement of fine aggregates with sugarcane fibres of grain size less than $4.75 \mathrm{~mm}$ was $0.1 \%$. The effect of sugarcane fibres on the durability of bacterial concrete is presented in this paper.To study the Stress -Strain behaviour of Sugarcane based Bacterial concrete (SBC), appropriate analytic SS model is developed that resembles the experimental behaviour of the various samples such as Conventional Concrete (CC), Bacterial Concrete (BC) and SBC. This work mainly targets on utilizing the earlier models and offers a new SS model that can well represent the actual SS behaviour of SBC samples. After finding the SS behaviour of $\mathrm{CC}, \mathrm{BC}$ and SBC specimens experimentally, equations are developed to characterise axial SS behaviour of CC, BC and SBC samples. From these mathematical equations, theoretical stress for CC, BC and SBC are calculated and compared with test values. The proposed equations have exposed good connection with test values authorizing the mathematical model developed.
\end{abstract}

Keywords: Bacterial concrete, Stress-Strain curves, Saenz model, Bacillus Subtilis..

\section{Introduction}

Concrete is generally used as a building material because it is readily available and cheap. The durability of concreteneeds tobecheckedformanyreasonsnamelytheexpansionofreinforcement bars, freezing and thawing effects, physical damage, chemical damage andcrack formation.Formationofcracksisaninevitablequalityofconcretestructures.Crack formation in concrete structures is the reason for strength loss. They offer substances such as chlorides, carbon dioxide, oxygen and water to enter into it which leads to corrosion. Healing of cracks is necessary to strengthen the concrete structures and to increase the life span and to serve the purpose for which it is intended. Bacterial concrete is a new invention of crack healing in an environmentally friendly manner without human intervention. The cracks are sealed by biologically produced calcium precipitate. A gram positive, rod shaped with tough protective endospore, Bacillus subtilis is used. It can remain viable for decades. In this study, sugarcane fibres are used with bacterial concrete to enhance the biological process of bacteria. Sugarcane fibres increase crack control, ductility and reduce environmental pollution.

For many years, researchers have developed mathematical models for SS relationships to define the nature of concrete in compression. The SS model is a reliable tool to estimate the strength, elongation, contraction and shear behaviour of concrete structural members like beams, columns and slabs. The compressive SS behaviour of concrete is a noteworthy subject in the flexural analysis of RC beams and columns and for exploring the ductility of concrete. The quantity of energy absorbed can also be obtained by calculating the total area under the SS curve.

Figure 1 shows the SS curve of a ductile material. The stress and strain values obtained from the cylinder compressive strength test are plotted here. It is noted from the graph that, the curve does not follow any specific pattern.

Figure 2 shows the SS curve for cement paste and aggregates individually and it is a linear curve. But for concrete, the internal crack formation results in non-linearity of SS curve. After conducting cylinder compressive strength tests for various concrete samples such as CC, BC and SBC. the SS behaviour of each sample was analysed. A mathematical model is suggested to confirm the experimental values against the analytical values for $\mathrm{CC}, \mathrm{BC}$ and SBC specimens. 

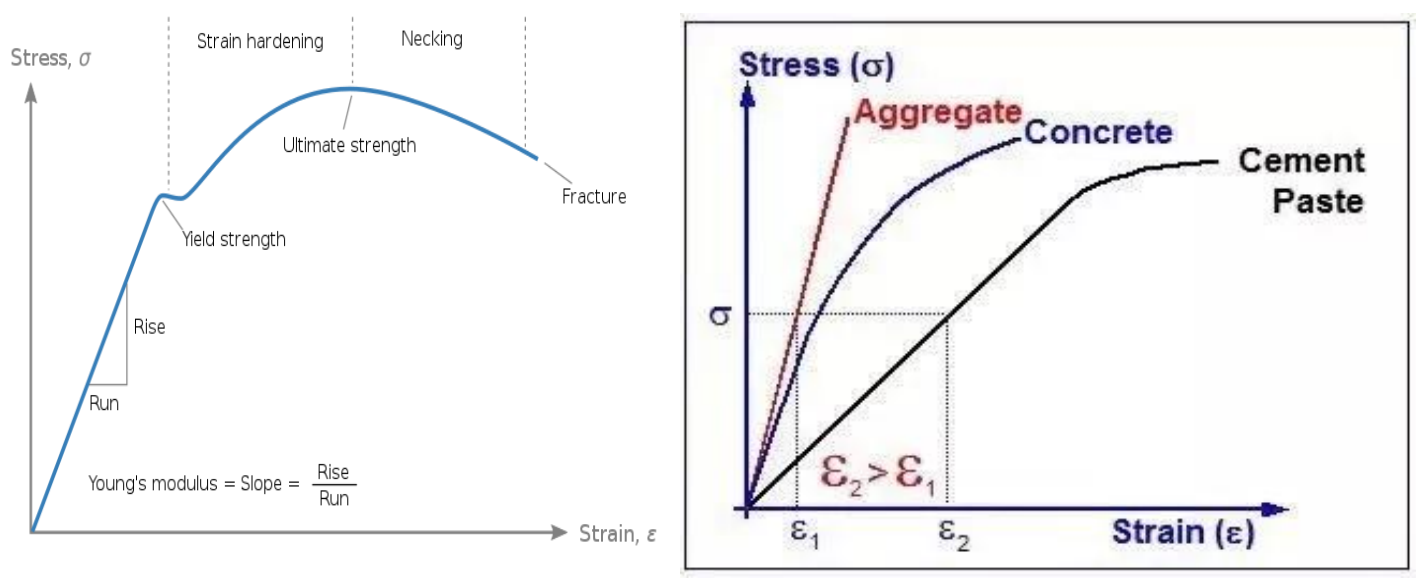

Figure 1SS curve of a Ductile Material. Figure 2 SS curve of cement and aggregates

\subsection{Types of Stress and Strain}

The types of stress are Normal stress, Shear stress or combination of both. In addition, they can be Uniaxial stress, Biaxial stress and Multi-axial stress. The forms of strain deformation are elongation, contraction, twisting and rotation.

\subsection{Types of SS curves}

If the stress and strain are calculated using original cross-section and gauge length, then they are designated as Engineering stress and Engineering strain. The curve drawn with these values is named as Engineering stressstrain curve shown in figure 3. If the stress and strain are derived from the reduced area at the time of failure of the specimen, then they are called True stress and True strain. This stage in the SS curve reveals its behaviour, which results in its mechanical properties. There are three stages in the SS curve, which are 1. Linear elastic region, 2. Strain hardening region and 3. Failure region. The area under the SS curve in Figure 4 is the Absorbed energy, which states the nature of ductility of the material.

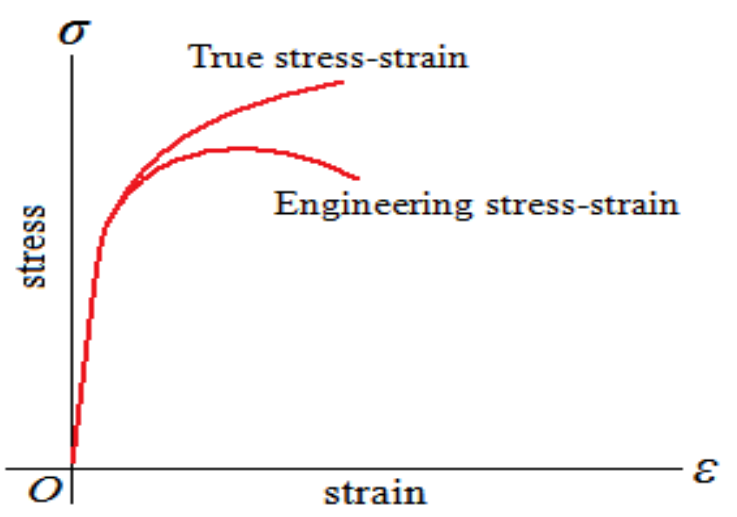

Figure 3 Engineering SSC and True SSC

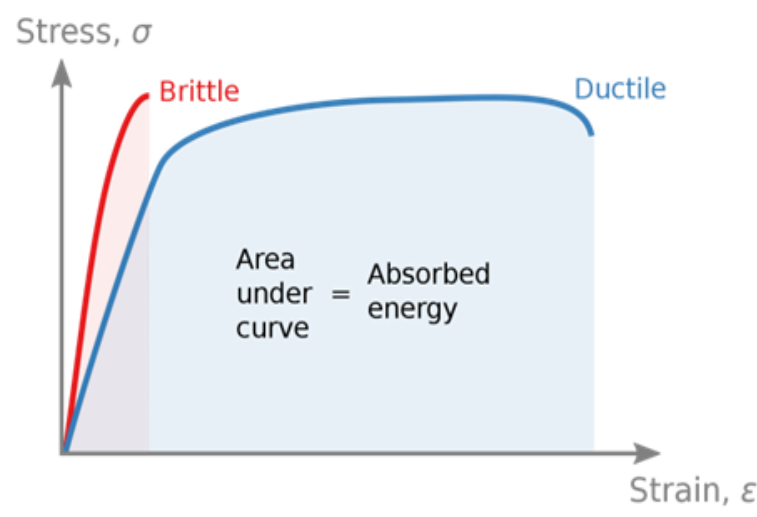

Figure 4 Area of Energy Absorption

\section{Methodology}

This paper mainly aims at developing the best aspects of earlier models and suggests a new SS model that denotes the SS behaviour of SBC. After attaining the SS behaviour of CC, BC and SBC experimentally, mathematical equations were developed to signify the axial SS behaviour of $\mathrm{CC}, \mathrm{BC}$ and SBC mixes. From these equations, analytical stress for $\mathrm{CC}, \mathrm{BC}$ and SBC were evaluated and compared with test values. The suggested equations have shown better correlation with experimental values, validating the mathematical model formed.

\section{Experimental Procedure}

In this work, SS behaviour of CC, BC and SBC specimens of strength grade M25 were studied. Cylindrical specimens of size $150 \mathrm{~mm}$ diameter X $300 \mathrm{~mm}$ heights were cast. There were 3 cylinders made of CC, 3 cylinders of BC and 3 cylinders of SBC. The tests were carried out on each of these specimens. All specimens were subjected to an axial compression as per IS: 516 -1999 to analyse the stress-strain characteristics. The experimental set up was shown in figure 5. 

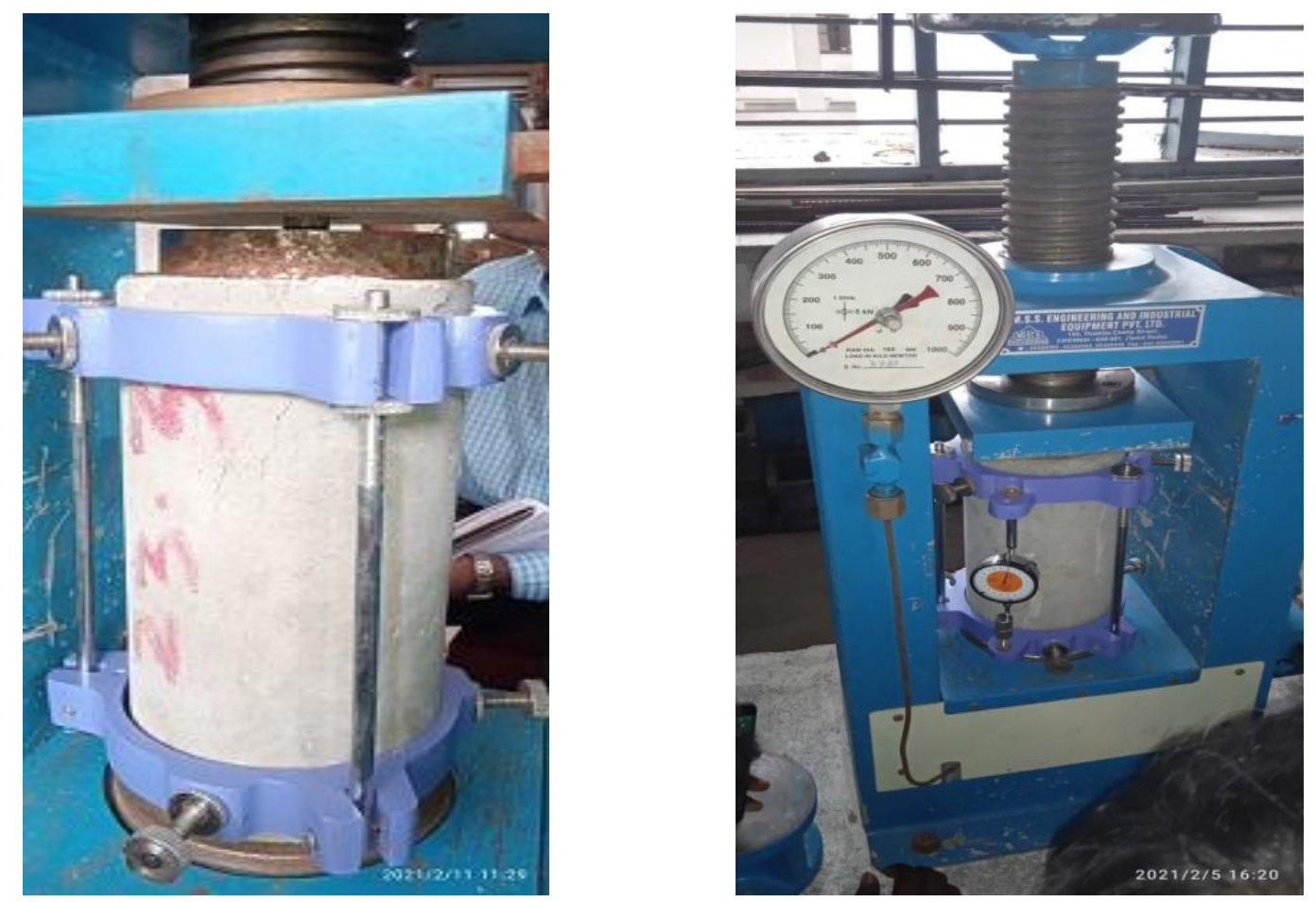

Figure 5 Experimental Setup

\section{Mathematical Modelling for SS Performance}

Researchers have developed mathematical models for predicting the SS behaviour of concrete. Some models are stated below.

Table 1Various Mathematical models

\begin{tabular}{|c|c|c|c|c|}
\hline Models & Year & Curve Equation & Region & Remarks \\
\hline Hognestad & 1951 & $\begin{array}{c}f=f_{0}\left[2 \frac{\varepsilon}{\varepsilon_{0}}-\left(\frac{\varepsilon}{\varepsilon_{0}}\right)^{2}\right] \\
f=f_{0}\left[1-0.15\left(\frac{\varepsilon-\varepsilon_{0}}{\varepsilon_{u}-\varepsilon_{0}}\right)\right]\end{array}$ & Descending & \\
\hline $\begin{array}{l}\text { Desayi \& } \\
\text { Krishnan }\end{array}$ & 1964 & $f=\frac{E_{0} \varepsilon}{1+\left(\varepsilon / \varepsilon_{0}\right)^{2}}$ & $\begin{array}{l}\text { Ascending and } \\
\text { Descending }\end{array}$ & $\begin{array}{l}\left(E_{0} / E_{s}\right) \text { Should } \\
\text { equal to } 2\end{array}$ \\
\hline Saenz & 1964 & $f=\frac{E_{0} \varepsilon}{1+\left(E_{0} / E_{s}-2\right)\left(\left(\varepsilon / \varepsilon_{0}\right)+\left(\varepsilon / \epsilon_{0}\right)^{2}\right.}$ & & $\begin{array}{l}\left(E_{0} / E_{s}\right) \quad \text { Should } \\
\text { equal or greater than }\end{array}$ \\
\hline Wang et al & 1978 & $f=f_{0}\left[\frac{A\left(\varepsilon / \varepsilon_{0}\right)+B\left(\varepsilon / \varepsilon_{0}\right)^{2}}{1+C\left(\varepsilon / \varepsilon_{0}\right)+D\left(\varepsilon / \varepsilon_{0}\right)^{2}}\right]$ & $\begin{array}{l}\text { Ascending } \\
\mathrm{A}=1.300501 \\
\mathrm{~B}=-0.835818 \\
\mathrm{C}=-0.699498 \\
\mathrm{D}=0.1641812\end{array}$ & $\begin{array}{l}\text { Descending } \\
A=0.349777 \\
B=-0.104963 \\
C=-1.650222 \\
D=0.895036\end{array}$ \\
\hline $\begin{array}{l}\text { Carreira \& } \\
\text { Chu }\end{array}$ & 1985 & $f=\frac{A\left(\varepsilon / \varepsilon_{0}\right) f_{0}}{A-1+\left(\varepsilon / \varepsilon_{0}\right)^{A}}$ & $\begin{array}{l}\text { Ascending and } \\
\text { Descending }\end{array}$ & $A=\frac{1}{1-\left(E_{s} / E_{o}\right)}$ \\
\hline Thanoon & 1997 & $f=f_{0}\left[\frac{A\left(\varepsilon / \varepsilon_{0}\right)}{\left(\varepsilon / \varepsilon_{0}\right)^{3}+B\left(\varepsilon / \varepsilon_{0}\right)^{2}+C\left(\varepsilon / \epsilon_{0}\right)+D}\right]$ & $\begin{array}{l}\text { Ascending and } \\
\text { Descending }\end{array}$ & $\begin{array}{l}\text { For Plain Concrete } \\
A=1.10 ; B=-1.30 \\
C=0.75 ; D=0.65\end{array}$ \\
\hline
\end{tabular}


Table 2Details of Notations

f

$f_{0}$

$\varepsilon_{\mathrm{o}}$

$\varepsilon_{\mathrm{u}}$

$\mathrm{E}_{\mathrm{o}}$

$\mathrm{E}_{\mathrm{s}}$

A, B, C and D
Stress Corresponding to the strain $\varepsilon$

Maximum Compressive Stress

Strain Corresponding to maximum Stress

Ultimate Strain

Initial Tangent Modulus at the Origin

Secant Modulus at the peak $\left(f_{0} / \varepsilon_{0}\right)$

Constants

\subsection{Model for SS behaviour of SBC}

Each model was checked with the observed SS values. The experimental data was correlated with Hognestad model, Wang et al model and modified Saenz 's model equations. The normalised stress and strain were calculated for each of the concrete samples. In Saenz's model equation, the constants A, B and C are identified in the ascending portion of the SS curve and D, E and F in the descending portion of the curve. The constants were found by applying the boundary conditions. There were four boundary conditions applied for the Saenz's model equation.

The Hognestad model was also checked with the experimental SS values. It was found that the experimental values were observed to be too different from the theoretical values for the elastic region and also for the failure region. In the middle region, the experimental and theoretical values were found to match.

In case of Wang et al model, the experimental values were in good correlation with theoretical values for the ascending portion and not for the descending portion.

The third trial was done with the Modified Saenz's equation. The equations for the both portions of theoretical SS curve are given below.

$$
\begin{aligned}
& \mathrm{y}=\frac{A x}{\left(1+B x+C x^{2}\right)}(\text { Ascending portion of the SSC) ----------- (1) } \\
& \mathrm{y}=\frac{D x}{\left(1+E x+F x^{2}\right)} \text { (Descending portion of the SSC) -------------(2) }
\end{aligned}
$$

Where $\mathrm{y}$ is the stress at any point; $\mathrm{x}$ is the corresponding strain at that point; similarly, the equations for ascending and descending portions of normalised SS curve are given below

$$
\begin{aligned}
& f / f_{0}=\frac{A^{\prime}\left(\varepsilon / \varepsilon_{0}\right)}{\left(1+B^{\prime}\left(\varepsilon / \varepsilon_{o}\right)+C^{\prime}\left(\varepsilon / \varepsilon_{o}\right)^{2}\right)}-1(3) \\
& f / f_{0}=\frac{D^{\prime}\left(\varepsilon / \varepsilon_{0}\right)}{\left(1+E^{\prime}\left(\varepsilon / \varepsilon_{o}\right)+F^{\prime}\left(\varepsilon / \varepsilon_{o}\right)^{2}\right)}-\text {--------(4) }
\end{aligned}
$$

The values of A', B', C', D', E' and F' were calculated by applying the boundary conditions. They are,

1. The ratio of SS ratio is zero at the origin; $\left(\varepsilon / \varepsilon_{0}\right)=0 ;\left(f / f_{0}\right)=0$

2. The strain ratio as well as the stress ratio at the peak is unity; $\left(\varepsilon / \varepsilon_{0}\right)=1 ;\left(f / f_{0}\right)=1$

3.The slope of the theoretical SS curve is zero; $\left(\varepsilon / \varepsilon_{0}\right)=1, d\left(f / f_{0}\right) / d\left(\varepsilon / \varepsilon_{0}\right)=0$

4. Record the Strain when $f / f_{0}=0.85$.

The values of A, B, C, D, E and F were calculated by using the equations given below. They are,

$$
\begin{aligned}
& A=A^{\prime}\left(f_{0} / \varepsilon_{0}\right), B=B^{\prime}\left(1 / \varepsilon_{0}\right), C=C^{\prime}\left(1 / \varepsilon_{0}\right)^{2} \\
& D=D^{\prime}\left(f_{0} / \varepsilon_{0}\right), E=E^{\prime}\left(1 / \varepsilon_{0}\right), F=F^{\prime}\left(1 / \varepsilon_{0}\right)^{2}
\end{aligned}
$$




\subsection{Formation of Theoretical Equations.}

Table 3Constants for ascending and descending portions of non-dimensional SSC

\begin{tabular}{|c|c|c|c|c|c|c|}
\hline Sample & $\mathbf{A}^{\prime}$ & B' & C' & D' & E' & F' \\
\hline CC & 3 & 1 & 1 & 0.126 & -1.874 & 1 \\
\hline BC & 0.35 & -1.65 & 1 & 0.071 & -1.929 & 1 \\
\hline SBC & 0.65 & -1.35 & 1 & 0.037 & -1.963 & 1 \\
\hline
\end{tabular}

Table 4Peak Stress and its corresponding strain

\begin{tabular}{|c|c|c|c|c|c|}
\hline \multicolumn{2}{|c|}{ CC } & \multicolumn{2}{c|}{ BC } & \multicolumn{2}{c|}{ SBC } \\
\hline $\mathbf{f}_{\mathbf{0}}$ & $\boldsymbol{\varepsilon}_{\mathbf{0}}$ & $\mathbf{f}_{\mathbf{o}}$ & $\boldsymbol{\varepsilon}_{\mathbf{0}}$ & $\mathbf{f}_{\mathbf{o}}$ & $\boldsymbol{\varepsilon}_{\mathbf{0}}$ \\
\hline 20.09 & 0.00065 & 21.22 & 0.0006 & 25.46 & 0.000353 \\
\hline
\end{tabular}

Table 5Ascending and Descending Portions Constants for Theoretical SS Curve

\begin{tabular}{|c|c|c|c|c|c|}
\hline \multicolumn{7}{|c|}{ Conventional Concrete } \\
\hline A & B & C & D & E & F \\
\hline 92723.08 & 1538.46 & 2366863.91 & 3894.37 & -2883.08 & 2366863.91 \\
\hline \multicolumn{7}{|c|}{ Bacterial Concrete } \\
\hline A & B & C & D & E & F \\
\hline 12378.33 & -2750.00 & 2777777.78 & 2511.03 & -3215.00 & 2777777.78 \\
\hline & Sugarcane Fibres Based Bacterial Concrete & F \\
\hline A & B & C & D & E & 8025102.52 \\
\hline 46881.02 & -3824.36 & 8025102.52 & 2668.61 & -5560.91 & \\
\hline
\end{tabular}

\subsection{Assessment of Theoretical Stress using proposed Mathematical Equations}

The Engineering SS, True SS and Normalised SS for CC,BC and SBC samples are tabulated in Tables 6,7 and 8 respectively. Figure 6 shows the SS curves of CC, BC and SBC. Theoretical stress have been found using proposed mathematical equations for $\mathrm{CC}, \mathrm{BC}$ and $\mathrm{SBC}$ which are resulting from modified Saenz's model. After developing the equations for SS curves of $\mathrm{CC}, \mathrm{BC}$ and $\mathrm{SBC}$ theoretical values of stress were calculated for each strain value and are tabulated in Tables 9,10 and 11 for CC, BC and SBC . Figure 7 shows the experimental and theoretical SS curves. Figure 8 shows the normalised experimental and theoretical SS curves.The theoretical SS curves were compared with experimental SS curves and found that, a good correlation was found with experimental SS curves for all samples of CC, BC and SBC.

\subsection{Determination of Modulus of Elasticity, Secant Modulus and Initial Tangent Modulus}

The static modulus of elasticity, $\mathrm{E}_{\mathrm{c}}$, the secant modulus (35-45\% of the maximum stress) and initial tangent modulus (the slope of the tangent drawn at the origin of the SS curve) were determined from the stress-strain curve. Table 12 shows the modulus of elasticity of concrete, secant and initial tangent moduli for CC, BC and SBC samples. 
Table 6Engineering Stress and Strains and True Stress and Strains for CC Samples

\begin{tabular}{|c|c|c|c|c|c|c|}
\hline \multicolumn{7}{|l|}{ CC SAMPLE } \\
\hline Strain in $\mathrm{mm}$ & $\begin{array}{l}\text { Stress in } \\
\mathrm{N} / \mathrm{mm}^{2}\end{array}$ & $\begin{array}{l}\text { True Strain } \\
\mathrm{mm}\end{array}$ & $\begin{array}{l}\text { True Stress } \\
\mathrm{N} / \mathrm{mm}^{2}\end{array}$ & $\begin{array}{l}\text { Eff. Plastic } \\
\text { strain } \\
\mathrm{mm}\end{array}$ & $\begin{array}{l}\text { Normalised } \\
\text { Strain } \\
\mathrm{mm}\end{array}$ & $\begin{array}{l}\text { Normalised } \\
\text { Stress } \\
\mathrm{N} / \mathrm{mm}^{2}\end{array}$ \\
\hline 0 & 0 & 0 & 0 & 0 & 0.00 & 0.00 \\
\hline 0.00001 & 1.41 & 0.00001 & 1.41 & 0.00000 & 0.01 & 0.08 \\
\hline 0.00004 & 2.83 & 0.00004 & 2.83 & 0.00003 & 0.06 & 0.15 \\
\hline 0.00011 & 4.24 & 0.00011 & 4.24 & 0.00010 & 0.15 & 0.23 \\
\hline 0.00011 & 5.66 & 0.00011 & 5.66 & 0.00011 & 0.16 & 0.31 \\
\hline 0.00013 & 7.07 & 0.00013 & 7.07 & 0.00013 & 0.19 & 0.39 \\
\hline 0.00017 & 8.49 & 0.00017 & 8.49 & 0.00016 & 0.23 & 0.46 \\
\hline 0.00017 & 9.90 & 0.00017 & 9.90 & 0.00016 & 0.24 & 0.54 \\
\hline 0.00022 & 11.32 & 0.00022 & 11.32 & 0.00021 & 0.31 & 0.62 \\
\hline 0.00025 & 12.73 & 0.00025 & 12.74 & 0.00024 & 0.35 & 0.69 \\
\hline 0.00029 & 13.58 & 0.00029 & 13.59 & 0.00028 & 0.40 & 0.74 \\
\hline 0.00031 & 14.15 & 0.00031 & 14.15 & 0.00030 & 0.43 & 0.77 \\
\hline 0.00032 & 16.98 & 0.00032 & 16.98 & 0.00031 & 0.45 & 0.93 \\
\hline 0.00032 & 18.39 & 0.00032 & 18.40 & 0.00032 & 0.46 & 1.00 \\
\hline 0.00033 & 18.96 & 0.00033 & 18.96 & 0.00032 & 0.46 & 1.03 \\
\hline 0.00065 & 20.09 & 0.00065 & 20.10 & 0.00065 & 0.92 & 1.10 \\
\hline 0.00071 & 18.32 & 0.00235 & 20.40 & 0.00235 & 1.00 & 1.00 \\
\hline 0.00079 & 16.10 & 0.00290 & 18.40 & 0.00289 & 1.11 & 0.88 \\
\hline 0.00085 & 14.32 & 0.00313 & 17.71 & 0.00312 & 1.20 & 0.78 \\
\hline 0.00091 & 11.20 & 0.00330 & 15.60 & 0.00329 & 1.28 & 0.61 \\
\hline
\end{tabular}

Table 7Engineering Stress and Strains and True Stress and Strain for BC Samples

\begin{tabular}{|l|l|l|l|l|l|l|}
\hline \multicolumn{2}{|l|}{ BC SAMPLES } & Strain in mm & $\begin{array}{l}\text { Stress } \\
\text { N/mm }\end{array}$ \\
\hline & 0 & $\begin{array}{l}\text { True Strain } \\
\mathrm{mm}\end{array}$ & $\begin{array}{l}\text { True Stress } \\
\text { N/mm }\end{array}$ & $\begin{array}{l}\text { Eff. Plastic } \\
\text { strain } \\
\mathrm{mm}\end{array}$ & $\begin{array}{l}\text { Normalised } \\
\text { Strain } \\
\mathrm{mm}\end{array}$ & $\begin{array}{l}\text { Normalised } \\
\text { Stress } \\
\text { N/mm }\end{array}$ \\
\hline 0 & 0 & 0 & 0 & 0.000 & 0.000 \\
\hline 0.000027 & 1.41 & 0.000027 & 1.41 & -0.000027 & 0.042 & 0.073 \\
\hline 0.000053 & 2.83 & 0.000053 & 2.83 & 0.000000 & 0.083 & 0.146 \\
\hline 0.000073 & 4.24 & 0.000073 & 4.24 & 0.000020 & 0.115 & 0.219 \\
\hline 0.000093 & 5.66 & 0.000093 & 5.66 & 0.000040 & 0.146 & 0.291 \\
\hline 0.000127 & 7.07 & 0.000127 & 7.07 & 0.000073 & 0.198 & 0.364 \\
\hline 0.000153 & 8.49 & 0.000153 & 8.49 & 0.000100 & 0.240 & 0.437 \\
\hline 0.000187 & 9.90 & 0.000187 & 9.90 & 0.000133 & 0.292 & 0.510 \\
\hline 0.000233 & 11.32 & 0.000233 & 11.32 & 0.000180 & 0.365 & 0.583 \\
\hline 0.000267 & 12.73 & 0.000267 & 12.74 & 0.000213 & 0.417 & 0.656 \\
\hline 0.000320 & 14.15 & 0.000320 & 14.15 & 0.000267 & 0.500 & 0.728 \\
\hline 0.000363 & 15.56 & 0.000363 & 15.57 & 0.000310 & 0.568 & 0.801 \\
\hline 0.000413 & 16.98 & 0.000413 & 16.98 & 0.000360 & 0.646 & 0.874 \\
\hline 0.000487 & 18.39 & 0.000487 & 18.40 & 0.000433 & 0.760 & 0.947 \\
\hline 0.000533 & 19.81 & 0.000533 & 19.82 & 0.000480 & 0.833 & 1.020 \\
\hline
\end{tabular}




\begin{tabular}{|l|l|l|l|l|l|l|}
0.000600 & 21.22 & 0.000600 & 21.23 & 0.000546 & 0.938 & 1.093 \\
\hline 0.000640 & 19.42 & 0.000640 & 19.43 & 0.000586 & 1.000 & 1.000 \\
\hline 0.000689 & 17.42 & 0.000689 & 17.43 & 0.000635 & 1.077 & 0.897 \\
\hline 0.000701 & 15.33 & 0.000701 & 15.34 & 0.000647 & 1.095 & 0.789 \\
\hline 0.000723 & 15.10 & 0.000723 & 15.11 & 0.000669 & 1.130 & 0.778 \\
\hline
\end{tabular}

Table 8Engineering Stress and Strains and True Stress and Strain for SBC Samples

\begin{tabular}{|c|c|c|c|c|c|c|}
\hline \multicolumn{7}{|c|}{ SBC SAMPLES } \\
\hline Strain in $\mathrm{mm}$ & $\begin{array}{l}\text { Stress in } \\
\mathrm{N} / \mathrm{mm}^{2}\end{array}$ & $\begin{array}{l}\text { True Strain } \\
\mathrm{mm}\end{array}$ & $\begin{array}{l}\text { True Stress } \\
\mathrm{N} / \mathrm{mm}^{2}\end{array}$ & $\begin{array}{l}\text { Eff. Plastic } \\
\text { strain } \\
\text { mm }\end{array}$ & $\begin{array}{l}\text { Normalised } \\
\text { Strain } \\
\mathrm{mm}\end{array}$ & $\begin{array}{l}\text { Normalised } \\
\text { Stress } \\
\mathrm{N} / \mathrm{mm}^{2}\end{array}$ \\
\hline 0 & 0 & 0 & 0.00 & 0 & 0.000 & 0.00 \\
\hline 0.000080 & 1.41 & 0.00008 & 1.41 & 0.00000 & 0.226 & 0.06 \\
\hline 0.000107 & 2.83 & 0.00011 & 2.83 & 0.00003 & 0.302 & 0.11 \\
\hline 0.000133 & 4.24 & 0.00013 & 4.24 & 0.00005 & 0.377 & 0.17 \\
\hline 0.000147 & 5.66 & 0.00015 & 5.66 & 0.00007 & 0.415 & 0.22 \\
\hline 0.000167 & 7.07 & 0.00017 & 7.07 & 0.00009 & 0.472 & 0.28 \\
\hline 0.000173 & 8.49 & 0.00017 & 8.49 & 0.00009 & 0.491 & 0.33 \\
\hline 0.000197 & 9.90 & 0.00020 & 9.90 & 0.00012 & 0.557 & 0.39 \\
\hline 0.000200 & 11.32 & 0.00020 & 11.32 & 0.00012 & 0.566 & 0.44 \\
\hline 0.000200 & 12.73 & 0.00020 & 12.73 & 0.00012 & 0.566 & 0.50 \\
\hline 0.000203 & 14.15 & 0.00020 & 14.15 & 0.00012 & 0.575 & 0.56 \\
\hline 0.000213 & 15.56 & 0.00021 & 15.57 & 0.00013 & 0.604 & 0.61 \\
\hline 0.000220 & 16.98 & 0.00022 & 16.98 & 0.00014 & 0.623 & 0.67 \\
\hline 0.000220 & 18.39 & 0.00022 & 18.40 & 0.00014 & 0.623 & 0.72 \\
\hline 0.000223 & 19.81 & 0.00022 & 19.81 & 0.00014 & 0.632 & 0.78 \\
\hline 0.000237 & 21.22 & 0.00024 & 21.23 & 0.00016 & 0.670 & 0.83 \\
\hline 0.000287 & 22.64 & 0.00029 & 22.64 & 0.00021 & 0.811 & 0.89 \\
\hline 0.000347 & 24.90 & 0.00035 & 24.91 & 0.00027 & 0.981 & 0.98 \\
\hline 0.000353 & 25.46 & 0.00035 & 25.47 & 0.00027 & 1.000 & 1.00 \\
\hline 0.000458 & 23.10 & 0.00046 & 23.11 & 0.00038 & 1.296 & 0.91 \\
\hline 0.000518 & 17.32 & 0.00052 & 17.33 & 0.00044 & 1.466 & 0.68 \\
\hline 0.000558 & 15.30 & 0.00056 & 15.31 & 0.00048 & 1.579 & 0.60 \\
\hline 0.000598 & 12.40 & 0.00060 & 12.41 & 0.00052 & 1.692 & 0.49 \\
\hline 0.000638 & 10.10 & 0.00064 & 10.11 & 0.00056 & 1.806 & 0.40 \\
\hline
\end{tabular}




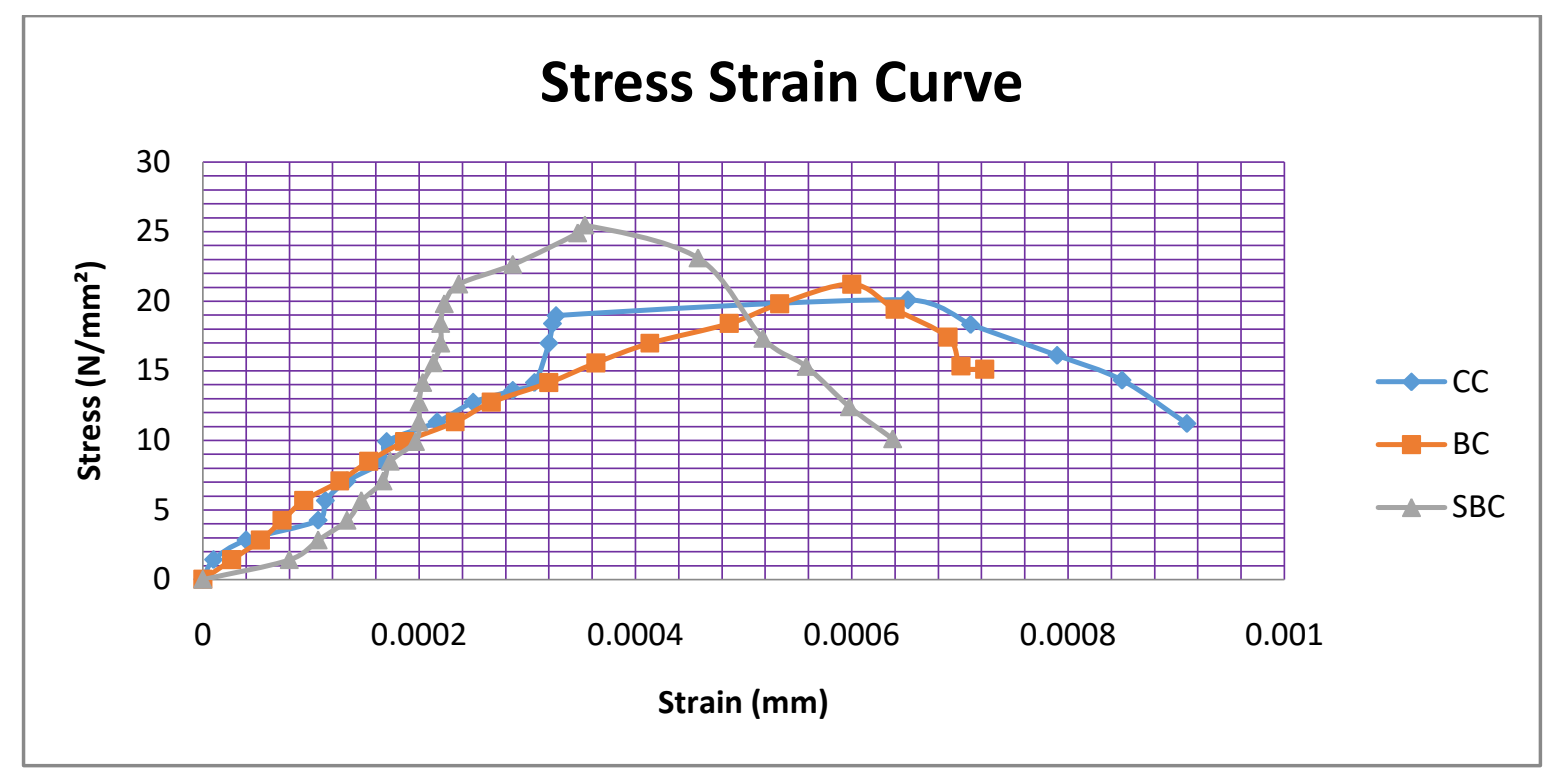

Figure 6 SS curve of CC, BC and SBC samples

Table 9 Experimental and Theoretical SS values for CC samples

\begin{tabular}{|c|c|c|c|c|c|}
\hline \multicolumn{6}{|c|}{ CC SAMPLES } \\
\hline Strain in $\mathrm{mm}$ & $\begin{array}{l}\text { Experimental } \\
\text { Stress } \\
\mathrm{N} / \mathrm{mm}^{2}\end{array}$ & $\begin{array}{l}\text { Theoretical } \\
\text { Stress } \\
\mathrm{N} / \mathrm{mm}^{2}\end{array}$ & $\begin{array}{l}\text { Normalised } \\
\text { Strain } \\
\mathrm{mm}\end{array}$ & $\begin{array}{l}\text { Normalised } \\
\text { Experimental } \\
\text { Stress } \\
\mathrm{N} / \mathrm{mm}^{2}\end{array}$ & $\begin{array}{l}\text { Normalised } \\
\text { Theoretical } \\
\text { Stress } \\
\mathrm{N} / \mathrm{mm}^{2}\end{array}$ \\
\hline 0 & 0 & 0.00 & 0.00 & 0.00 & 0.00 \\
\hline 0.00001 & 1.41 & 0.91 & 0.02 & 0.07 & 0.05 \\
\hline 0.00004 & 2.83 & 3.48 & 0.06 & 0.14 & 0.17 \\
\hline 0.00011 & 4.24 & 8.30 & 0.16 & 0.21 & 0.41 \\
\hline 0.00011 & 5.66 & 8.72 & 0.17 & 0.28 & 0.43 \\
\hline 0.00013 & 7.07 & 9.91 & 0.20 & 0.35 & 0.49 \\
\hline 0.00017 & 8.49 & 11.69 & 0.26 & 0.42 & 0.58 \\
\hline 0.00017 & 9.90 & 11.85 & 0.26 & 0.49 & 0.59 \\
\hline 0.00022 & 11.32 & 13.91 & 0.33 & 0.56 & 0.69 \\
\hline 0.00025 & 12.73 & 15.13 & 0.38 & 0.63 & 0.75 \\
\hline 0.00029 & 13.58 & 16.25 & 0.44 & 0.68 & 0.81 \\
\hline 0.00031 & 14.15 & 16.78 & 0.47 & 0.70 & 0.84 \\
\hline 0.00032 & 16.98 & 17.10 & 0.49 & 0.85 & 0.85 \\
\hline 0.00032 & 18.39 & 17.18 & 0.50 & 0.92 & 0.86 \\
\hline 0.00033 & 18.96 & 17.26 & 0.50 & 0.94 & 0.86 \\
\hline 0.00065 & 20.09 & 20.09 & 1.00 & 1.00 & 1.00 \\
\hline 0.00071 & 18.32 & 18.92 & 1.09 & 0.91 & 0.94 \\
\hline 0.00079 & 16.10 & 15.42 & 1.21 & 0.80 & 0.77 \\
\hline 0.00085 & 14.32 & 12.76 & 1.30 & 0.71 & 0.64 \\
\hline 0.00091 & 11.20 & 10.53 & 1.40 & 0.56 & 0.52 \\
\hline
\end{tabular}

Table 10 Experimental and Theoretical non-dimensional SS valuesfor BC samples

\begin{tabular}{|l|l|l|l|l|l|}
\hline BC SAMPLES & Experimental \\
Strain in $\mathrm{mm}$ & $\begin{array}{l}\text { Stress } \\
\mathrm{N} / \mathrm{mm}^{2}\end{array}$ & $\begin{array}{l}\text { Theoretical } \\
\text { Stress } \\
\mathrm{N} / \mathrm{mm}^{2}\end{array}$ & $\begin{array}{l}\text { Normalised } \\
\text { Strain } \\
\mathrm{mm}\end{array}$ & $\begin{array}{l}\text { Normalised } \\
\text { Experimental } \\
\text { Stress } \\
\mathrm{N} / \mathrm{mm}^{2}\end{array}$ & $\begin{array}{l}\text { Normalised } \\
\text { Theoretical } \\
\text { Stress } \\
\mathrm{N} / \mathrm{mm}^{2}\end{array}$ \\
\hline 0 & 0 & 0.00 & 0.000 & 0.00 & 0.00 \\
\hline 0.000027 & 1.41 & 0.36 & 0.042 & 0.07 & 0.02 \\
\hline 0.000053 & 2.83 & 0.77 & 0.083 & 0.13 & 0.04 \\
\hline
\end{tabular}




\begin{tabular}{|l|l|l|l|l|l|}
0.000073 & 4.24 & 1.12 & 0.115 & 0.20 & 0.05 \\
\hline 0.000093 & 5.66 & 1.51 & 0.146 & 0.27 & 0.07 \\
\hline 0.000127 & 7.07 & 2.25 & 0.198 & 0.33 & 0.11 \\
\hline 0.000153 & 8.49 & 2.95 & 0.240 & 0.40 & 0.14 \\
\hline 0.000187 & 9.90 & 3.96 & 0.292 & 0.47 & 0.19 \\
\hline 0.000233 & 11.32 & 5.67 & 0.365 & 0.53 & 0.27 \\
\hline 0.000267 & 12.73 & 7.11 & 0.417 & 0.60 & 0.34 \\
\hline 0.000320 & 14.15 & 9.79 & 0.500 & 0.67 & 0.46 \\
\hline 0.000363 & 15.56 & 12.24 & 0.568 & 0.73 & 0.58 \\
\hline 0.000413 & 16.98 & 15.14 & 0.646 & 0.80 & 0.71 \\
\hline 0.000487 & 18.39 & 18.85 & 0.760 & 0.87 & 0.89 \\
\hline 0.000533 & 19.81 & 20.41 & 0.833 & 0.93 & 0.96 \\
\hline 0.000600 & 21.22 & 21.22 & 0.938 & 1.00 & 1.00 \\
\hline 0.000640 & 19.42 & 20.04 & 1.000 & 0.92 & 0.94 \\
\hline 0.000689 & 17.42 & 16.71 & 1.077 & 0.82 & 0.79 \\
\hline 0.000701 & 15.33 & 15.82 & 1.095 & 0.72 & 0.75 \\
\hline 0.000723 & 15.10 & 14.23 & 1.130 & 0.71 & 0.67 \\
\hline
\end{tabular}

Table 11 Experimental and Theoretical non-dimensional SS valuesfor SBC samples

\begin{tabular}{|c|c|c|c|c|c|}
\hline \multicolumn{6}{|c|}{ SBC SAMPLES } \\
\hline Strain in mm & $\begin{array}{l}\text { Experimental } \\
\text { Stress } \\
\mathrm{N} / \mathrm{mm}^{2}\end{array}$ & $\begin{array}{l}\text { Theoritical } \\
\text { Stress } \\
\mathrm{N} / \mathrm{mm}^{2}\end{array}$ & $\begin{array}{l}\text { Normalised } \\
\text { Strain } \\
\mathrm{mm}\end{array}$ & $\begin{array}{l}\text { Normalised } \\
\text { Experimental } \\
\text { Stress } \\
\mathrm{N} / \mathrm{mm}^{2}\end{array}$ & $\begin{array}{l}\text { Normalised } \\
\text { Theoritical } \\
\text { Stress } \\
\mathrm{N} / \mathrm{mm}^{2}\end{array}$ \\
\hline 0 & 0 & 0.00 & 0.000 & 0.00 & 0.00 \\
\hline 0.000080 & 1.41 & 5.03 & 0.226 & 0.06 & 0.20 \\
\hline 0.000107 & 2.83 & 7.32 & 0.302 & 0.11 & 0.29 \\
\hline 0.000133 & 4.24 & 9.88 & 0.377 & 0.17 & 0.39 \\
\hline 0.000147 & 5.66 & 11.24 & 0.415 & 0.22 & 0.44 \\
\hline 0.000167 & 7.07 & 13.34 & 0.472 & 0.28 & 0.52 \\
\hline 0.000173 & 8.49 & 14.05 & 0.491 & 0.33 & 0.55 \\
\hline 0.000197 & 9.90 & 16.52 & 0.557 & 0.39 & 0.65 \\
\hline 0.000200 & 11.32 & 16.86 & 0.566 & 0.44 & 0.66 \\
\hline 0.000200 & 12.73 & 16.86 & 0.566 & 0.50 & 0.66 \\
\hline 0.000203 & 14.15 & 17.20 & 0.575 & 0.56 & 0.68 \\
\hline 0.000213 & 15.56 & 18.21 & 0.604 & 0.61 & 0.72 \\
\hline 0.000220 & 16.98 & 18.85 & 0.623 & 0.67 & 0.74 \\
\hline 0.000220 & 18.39 & 18.85 & 0.623 & 0.72 & 0.74 \\
\hline 0.000223 & 19.81 & 19.17 & 0.632 & 0.78 & 0.75 \\
\hline 0.000237 & 21.22 & 20.38 & 0.670 & 0.83 & 0.80 \\
\hline 0.000287 & 22.64 & 23.86 & 0.811 & 0.89 & 0.94 \\
\hline 0.000347 & 24.90 & 25.45 & 0.981 & 0.98 & 1.00 \\
\hline 0.000353 & 25.46 & 25.46 & 1.000 & 1.00 & 1.00 \\
\hline 0.000458 & 23.10 & 22.20 & 1.296 & 0.91 & 0.87 \\
\hline 0.000518 & 17.32 & 18.54 & 1.466 & 0.68 & 0.73 \\
\hline 0.000558 & 15.30 & 17.47 & 1.579 & 0.60 & 0.69 \\
\hline 0.000598 & 12.40 & 12.39 & 1.692 & 0.49 & 0.49 \\
\hline 0.000638 & 10.10 & 9.68 & 1.806 & 0.40 & 0.38 \\
\hline
\end{tabular}


Table 12 Modulus of Elasticity and Toughness

\begin{tabular}{|c|c|c|}
\hline Sample ID & $\begin{array}{c}\text { Elastic Modulus } \\
(\mathrm{GPa})\end{array}$ & $\begin{array}{c}\text { Toughness } \\
(\mathrm{MPa})\end{array}$ \\
\hline CC & 29.31 & 264 \\
\hline BC & 31.26 & 296 \\
\hline SBC & 32.13 & 310 \\
\hline
\end{tabular}

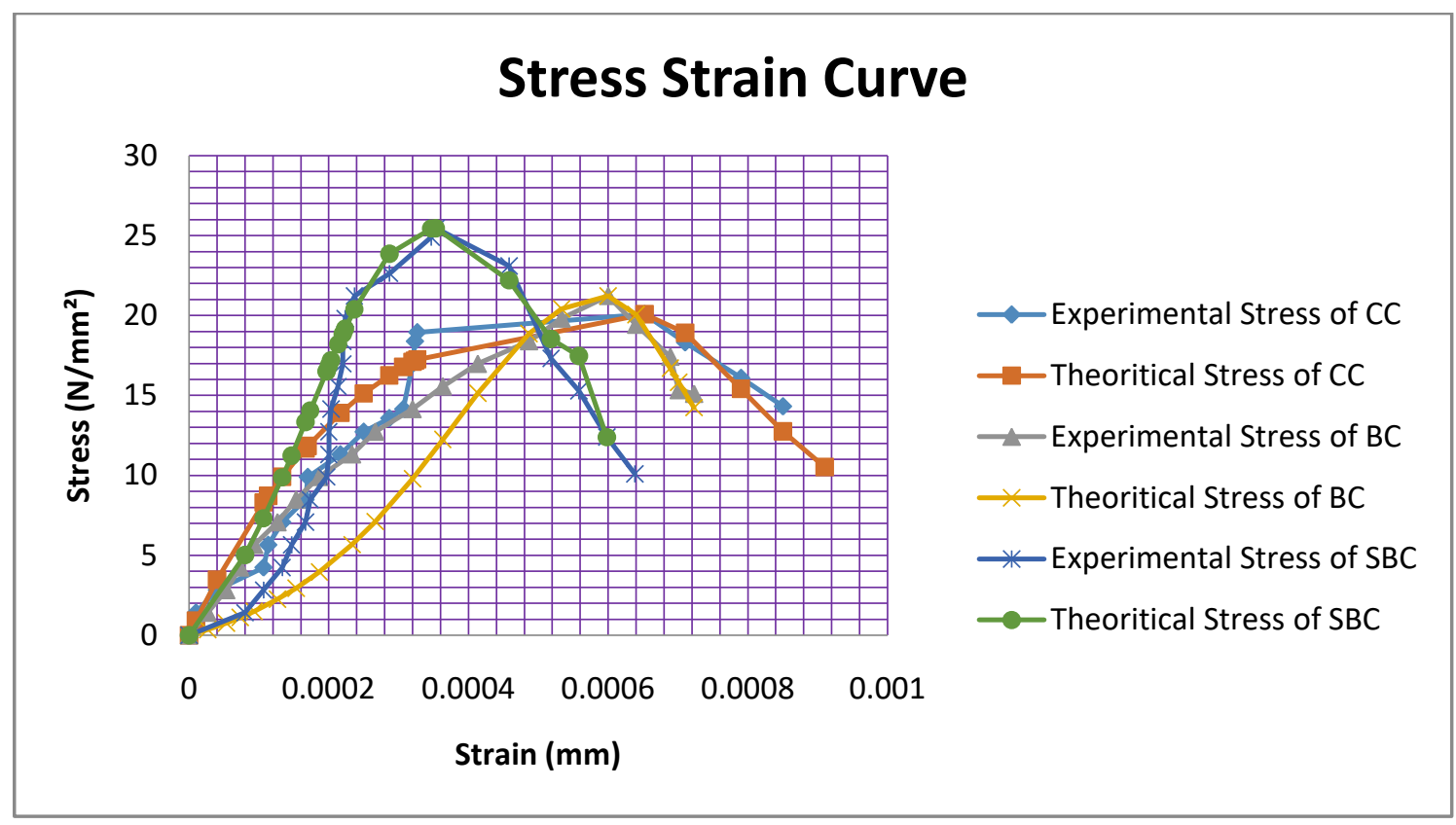

Figure 7 SS curves for various samples

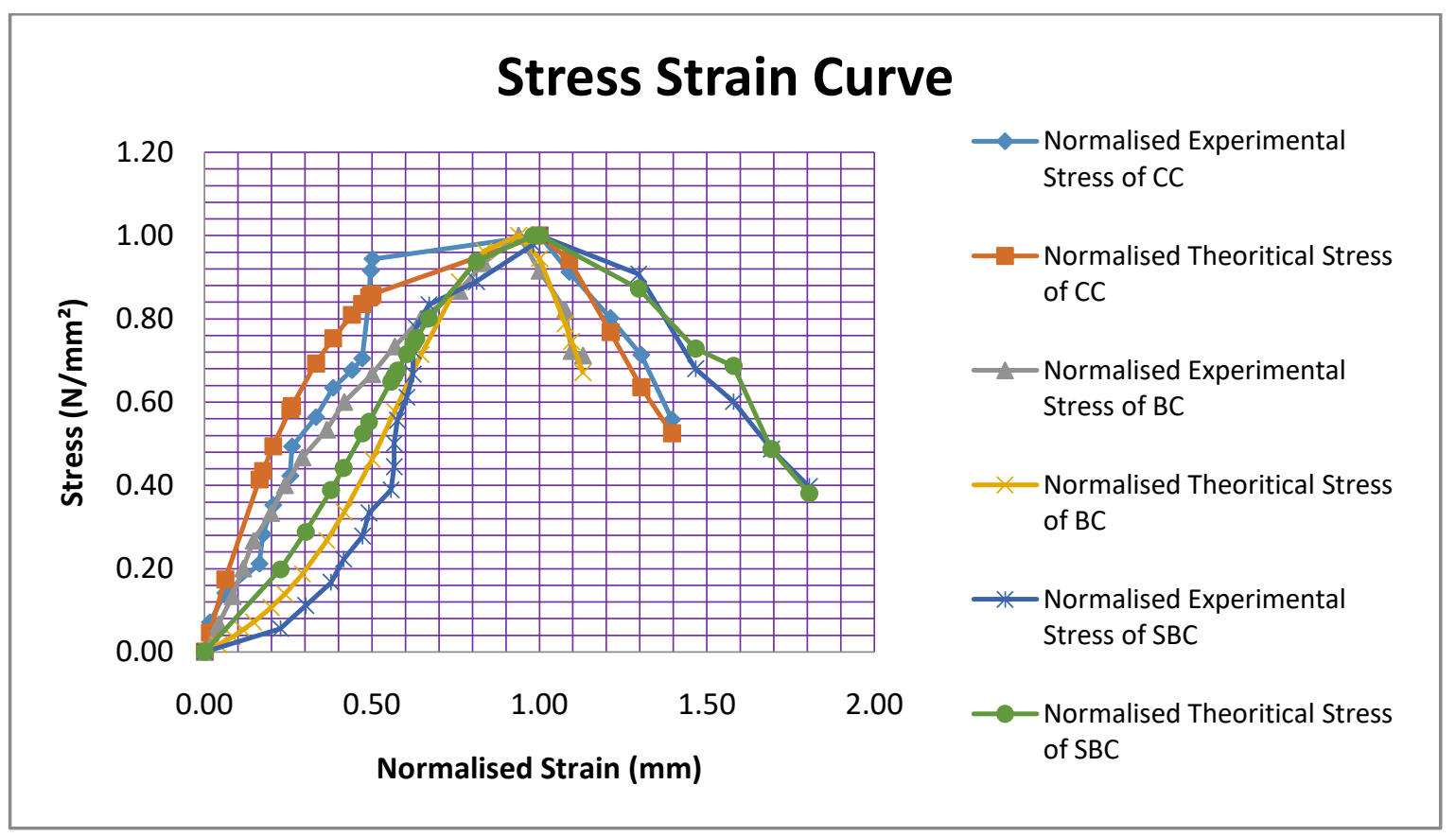

Figure 8 SS curves for various samples (Normalised values)

\section{Discussions}

The Engineering stress and strain, True stress and strain and Normalised stress and strain were tabulated for the samples CC, BC and SBC in the tables 6, 7 and 8.. The stress-strain curves for the CC, BC and SBC were shown in Figure 6. 


\subsection{SS curves}

Figure 6 denotes the SSC of M25 grade SBC. Replacement of small portion $(0.1 \%)$ of aggregate with Sugarcane fibres in BC has influence on the SSC of concrete. The shape of the SS curve for all the samples were different from each other. The Peak stress of SBC sample is $25.46 \mathrm{~N} / \mathrm{mm}^{2}$, ofBC sample is $21.22 \mathrm{~N} / \mathrm{mm}^{2}$ and for CC sample is $20.09 \mathrm{~N} / \mathrm{mm}^{2}$. The corresponding strains are $0.000353 \mathrm{~mm}$ for SBC samples, $0.0006 \mathrm{~mm}$ for BC samples and $0.00065 \mathrm{~mm}$ CC samples. It is found that the peak stress is maximum for SBC samples.

\subsection{Validation of suggested model}

The comparative analysis graph of experimental and theoretical SS values was represented in figure 7. The SSC of BC and CC were having similar curve shapes. But the shape of SBC is different. The Normalised experimental and theoretical SS curves are shown in figure 8. The Normalised theoretical values of each sample were in good correlation with its Normalised experimental values. The SBC samples have revealed improved stress values for the same strain levels compared to that of $\mathrm{CC}$ and $\mathrm{BC}$ samples.

\subsection{Modulus of Elasticity and Toughness}

Toughness of M25 grade SBC mix has revealed an increase of $4 \%$ and $17 \%$ when compared to same grade of $\mathrm{BC}$ and $\mathrm{CC}$ mixes.

\section{Conclusion}

From the test results obtained throughout this study, the following conclusions can be made:

1. The SBC samples have shown better stress values for the same strain levels compared to that of CC and BC samples.

2. The strain at peak stress of $\mathrm{CC}$ is $0.00065 \mathrm{~mm}$ and for $\mathrm{BC}$ is $0.00060 \mathrm{~mm}$. but the strain at peak stress for $\mathrm{SBC}$ is $0.000353 \mathrm{~mm}$.

3. The Mathematical equations for the SS values of CC, BC and SBC samples have been suggested in the form of $y=A x /(1+B x+C x 2)$, both for ascending and descending portions of the curves with different set of constants. The proposed equations have revealed that there is a good connection with test values.

4. The Saenz mathematical model was found to be best suited for analysis of the behaviour of SBC samples.

5. Toughness of SBC sample is increased by $4 \%$ when compared to BC and increased by $17 \%$ when compared to $\mathrm{CC}$.

\section{References}

ACI Committee 363, "State-of-the-Art Report on High Strength Concrete," ACI Journal, vol. 81, no. 4, pp. 364411, July-August, 1984.

Attard, M. M. \& Setunge, S. 1996. "SS relation-ship of confined and unconfined concrete." ACI Materials Journal 93(5): 432-442.

Carreira D, Chu K-H. "SS relationship for plain concrete in compression,” ACI Journal, No. 6, 82(1985)797-804.

Hsu L S, Hsu C T T 1994 “Complete stress-strain behaviour of high strength concrete under compression.” Mag. Concr. Res. 46: 301-312.

K. K. B. Dahl, "Uniaxial SS Curves for Normal and High Strength Concrete," ABK Report no. R282, Department of Structural Engineering, Technical University of Denmark, 1992.

M. M. Attard and S. Setunge, "SS Relationship of Confined and Unconfined Concrete," ACI Materials Journal, vol. 93, no. 5, pp. 432-442, September-October, 1996.

Madhu Karthik Murugesan Reddiar (2009) "SS Model Of Unconfined And Confined Concrete And Stress-Block Parameters," A MS Thesis, Texas A\&M University

P. T. Wang, S. P. Shah, and A. E. Naaman, "SS Curves of Normal and Lightweight Concrete in Compression," ACI Materials Journal, vol. 75, no. 11, pp. 603- 611, November-December, 1978.

Popovics, S. (1973). "A numerical approach to the complete SS curves of concrete." Cement and Concrete Research, 3(5), 583-599.

Saenz LP. Discussion of Paper by Desai P, Krishnan S. "Equation for SS curve of concrete," Journal of ACI, Proc., No. 9, 61(1964) 1229-35.

Thorenfeldt, E., Tomaszewicz, A., and Jensen, J.J. (1987). "Mechanical properties of highstrength concrete and application in design." Proc. of the Symposium on Utilization of High-Strength Concrete, Tapir, Trondheim, Norway, 149-159.

Tsai, W.T. (1988). "Uniaxial compression SS relation of concrete." J. Struct. Eng., 114(9), 2133-213 